\title{
Review Article \\ Clinical Use of Aspirin in Treatment and Prevention of Cardiovascular Disease
}

\author{
Yuxiang Dai and Junbo Ge \\ Shanghai Cardiovascular Institute and Department of Cardiology, Zhongshan Hospital, Fudan University, Shanghai 200032, China \\ Correspondence should be addressed to Junbo Ge, ge.junbo@zs-hospital.sh.cn \\ Received 10 June 2011; Accepted 17 October 2011 \\ Academic Editor: Christian Doutremepuich \\ Copyright ( $\odot 2012$ Y. Dai and J. Ge. This is an open access article distributed under the Creative Commons Attribution License, \\ which permits unrestricted use, distribution, and reproduction in any medium, provided the original work is properly cited. \\ Cardiovascular disease (CVD), principally heart disease and stroke, is the leading cause of death for both males and females in \\ developed countries. Aspirin is the most widely used and tested antiplatelet drug in CVD, and it is proven to be the cornerstone \\ of antiplatelet therapy in treatment and prevention of CVD in clinical trials in various populations. In acute coronary syndrome, \\ thrombotic stroke, and Kawasaki's disease, acute use of aspirin can decrease mortality and recurrence of cardiovascular events. As \\ secondary prevention, aspirin is believed to be effective in acute coronary syndrome, stable angina, revascularization, stroke, TIA, \\ and atrial fibrillation. Aspirin may also be used for patients with a high risk of future CVD for primary prevention, but the balance \\ between benefits and the possibility of side effects must be considered.
}

\section{Introduction}

Cardiovascular disease (CVD) continues to be the leading clinical and public health problem in developed countries and increasingly so throughout the world. Heart disease and stroke are the two main manifestations associated with CVD. The World Health Organization estimates that CVD will be the leading cause of death and disability worldwide by the year 2020 [1].

Millions of patients worldwide take low-dose aspirin on a daily basis for the treatment and prevention of CVD. By far, aspirin is the most widely tested antiplatelet drug in randomized trials of treatment and prevention of CVD [2]. Despite being one of the most widely used drugs in the 20th century, the benefits of aspirin in CVD have only relatively recently been recognized. This paper aims to provide clinical practice with a review of the evidence related to the use of aspirin for the treatment and prevention of cardiovascular events.

\section{Mechanism of Action}

Aspirin's mechanism of action involves inhibition of platelet activation and aggregation, which was first described in 1971 by British pharmacologist John Vane [3]. He demonstrated that the main mechanism of action was the irreversible inhibition of the platelet-dependent enzyme cyclooxygenase $(\mathrm{COX})$, thereby preventing the synthesis of prostaglandins. Subsequent researchers identified two COX isoenzymes, COX- 1 and COX-2 $[4,5]$. In platelets, the COX-1 enzyme produces thromboxane A2, a powerful promoter of platelet aggregation. Thus, aspirin, by irreversibly inactivating COX1 , thereby blocking the generation of thromboxane A2, derives a potential antiplatelet effect [6].

Platelet activation and aggregation with subsequent activation of the clotting cascade play critical roles in the onset of acute occlusive vascular events, such as MI and occlusive cerebrovascular accident (CVA) [7]. Because platelets do not have nucleus and thus cannot regenerate COX, they become an excellent target for antithrombotic therapy, while aspirin shows both immediate and long-term effects on platelets [8].

Other mechanisms of aspirin in CVD may also work. Aspirin blocks the formation of COX-dependent vasoconstrictors, which contribute to endothelial dysfunction in atherosclerosis [9]. Thus, improvement of endothelial dysfunction with aspirin may improve vasodilation, reduce thrombosis, and inhibit progression of atherosclerosis. Furthermore, aspirin reduces the inflammatory response in patients with coronary artery disease [10] and may inhibit 
the progression of atherosclerosis by protecting low-density lipoprotein from oxidation [11].

\section{Treatment in Cardiovascular Disease}

3.1. Therapy for Acute Coronary Syndrome. Convincing data support the use of aspirin in the acute treatment of acute coronary syndrome (ACS), including ST-elevation myocardial infarction (STEMI), non-ST-elevation myocardial infarction (NSTEMI) and unstable angina pectoris (UA) [12-14]. For ACS patients, the current American Heart Association/American College of Cardiology (AHA/ACC) guidelines recommend that aspirin should be administered as soon as possible with an initial loading dose of 162$325 \mathrm{mg}$ and continued indefinitely with a dose of 75-162 mg daily $[15,16]$. In the second International Study of Infarct Survival (ISIS-2) study, the use of aspirin (162 mg chewed, to ensure rapid therapeutic blood levels) was associated with a $23 \%$ reduction of vascular mortality rate in MI patients and close to a $50 \%$ reduction of nonfatal reinfarction or stroke, with benefits seen in both men and women [12]. In UA and USTEMI patients, aspirin has been shown to reduce the risk of fatal or nonfatal MI by $50-70 \%$ during the acute phase and by $50-60 \%$ at 3 months to 3 years $[13,14]$.

The highest benefit of aspirin was seen in those undergoing coronary angioplasty, with a 53\% $(P<0.0002)$ reduction in MI, stroke, or vascular deaths [17]. In percutaneous coronary intervention (PCI), the use of aspirin significantly reduces abrupt closure after balloon angioplasty and significantly reduces stent thrombosis rates [18].

3.2. Therapy for Kawasaki's Disease. Kawasaki's disease, which is a kind of acute vasculitis, occurs most commonly in children and in 15 to $25 \%$ of untreated cases results in the development of coronary artery aneurysms [19]. In the consensus guidelines from the Seventh American College of Chest Physicians (ACCP) Conference on Antithrombotic and Thrombolytic Therapy, high-dose aspirin (80$100 \mathrm{mg} / \mathrm{kg} /$ day) is recommended during the acute phase of the illness for its antiinflammatory effects, followed by lowdose aspirin (3-5 mg/kg/day) for its antiplatelet effect for 7 weeks or longer, maintaining it until the patient shows no evidence of coronary changes. In children with coronary aneurysms, long-term anticoagulation with warfarin and low-dose aspirin is recommended [20].

3.3. Therapy for Thromboembolic Stroke. With regard to stroke, the International Stroke Trial (IST) [21] and the Chinese Acute Stroke Trial (CAST) [22] together enrolled more than 40,000 patients admitted to hospital within 48 hours of the onset of stroke symptoms, who were randomized within 48 hours of the onset of symptoms to 2 to 4 weeks of daily aspirin therapy $(300 \mathrm{mg}$ and $160 \mathrm{mg}$, resp.) or placebo. Results from both trials suggest that aspirin therapy decreased the risk of recurrent stroke and death without significantly increasing the risk of hemorrhagic stroke $[21,22]$. These results are consistent with biochemical evidence of episodic platelet activation during the first $48 \mathrm{~h}$ after the onset of symptoms of an acute ischemic stroke and with suppression of in vivo TXA2 biosynthesis in patients receiving low-dose aspirin in this setting.

\section{Secondary Prevention}

Secondary prevention refers to the use of aspirin to prevent cardiovascular and cerebrovascular events in patients who have already experienced such an event or who have a high risk of an event. Long-term aspirin therapy reduces the yearly risk of serious vascular events (nonfatal myocardial infarction, nonfatal stroke, or vascular death), which corresponds to an absolute reduction of nonfatal events and to a smaller, but still definite, reduction in vascular death. Against these benefits, the absolute increase in major gastrointestinal or other major extracranial bleeds is relatively smaller. Hence, for secondary prevention, the benefits of aspirin therapy substantially exceed the risks, and aspirin is recommended as secondary prevention in conjunction with lifestyle change and stopping smoking to reduce an individual's overall risk of further cardiovascular events.

The Antithrombotic Trialists' (ATT) Collaboration performed a meta-analysis in 2002, which examined 287 randomized studies with 135000 high-risk patients in comparisons of antiplatelet therapy (predominantly aspirin) versus control and 77000 in comparisons of different antiplatelet regimens [17]. The results showed that among these highrisk patients, including acute $\mathrm{MI}$, acute stroke, previous stroke or transient ischemic attack (TIA), peripheral arterial disease, atrial fibrillation, antiplatelet therapy reduced the combined outcome of any serious vascular event by about $25 \%$, reduced nonfatal myocardial infarction by about $33 \%$, reduced nonfatal stroke by about $25 \%$, and reduced vascular mortality by about $17 \%$. In each of the high-risk categories, the absolute benefits outweighed the absolute risks of major extracranial bleeding.

For the choice of aspirin dosage, this analysis showed that COX is virtually completely inhibited in platelets, producing an antithrombotic effect, within a few days of beginning $75 \mathrm{mg}$ aspirin daily. It was indicated that high doses of 500$1500 \mathrm{mg}$ aspirin daily (which are more gastrotoxic48) were no more effective than medium doses of $160-325 \mathrm{mg} /$ day or low doses of 75-150 mg/day. Low-dose aspirin (75-150 mg daily) is an effective antiplatelet regimen for long-term use, and the effects of doses lower than $75 \mathrm{mg}$ daily were less certain. In clinical acute settings requiring an immediate antithrombotic effect (such as acute myocardial infarction, acute ischaemic stroke, unstable angina), an initial loading dose of about 150-300 mg aspirin should probably be given [17].

More recently, ATT Collaboration conducted another meta-analysis involving 16 secondary prevention trials (17000 individuals at high average risk, 43000 personyears, 3306 serious vascular events) that compared longterm aspirin versus control. This analysis showed that aspirin allocation yielded a greater absolute reduction in serious vascular events $(6.7 \%$ versus $8.2 \%$ per year, $P<0.0001)$, with a nonsignificant increase in haemorrhagic stroke but reductions of about $20 \%$ in total stroke (2.08\% versus $2.54 \%$ 
per year, $P=0.002)$ and in coronary events $(4.3 \%$ versus 5.3\% per year, $P<0.0001$ ) [23].

Aspirin (or another oral antiplatelet drug) is protective in most types of patient at increased risk of occlusive vascular events, including those with an acute myocardial infarction or ischaemic stroke, unstable or stable angina, previous myocardial infarction, stroke or cerebral ischaemia, peripheral arterial disease, or atrial fibrillation.

4.1. Secondary Prevention for Acute Coronary Syndromes. The benefit of aspirin therapy for preventing cardiovascular events in patients with ACS (STEMI, USTEMI, UP) has been definitively demonstrated in several trials [13, 14, 24, 25]. The previous meta-analysis by the ATT Collaboration [17] reviewed 18788 patients with a history of MI from the 12 most important randomized clinical trials of aspirin and showed that aspirin therapy reduced the relative risk of nonfatal MI by $28 \%(P<0.0001)$, vascular death by $15 \%$ $(P<0.0006)$, and overall mortality by $11 \%(P=0.02)$. The daily dosage of 80-325 $\mathrm{mg}$ appears to be effective in reducing the risk of cardiovascular events.

The 2007 ACC/AHA guidelines for the management of patients with unstable angina and non-ST-segment elevation MI recommend initiating daily aspirin therapy with at least $162 \mathrm{mg}$ as soon as possible after clinical presentation, with 75-325 mg daily indefinitely thereafter [15]. The 2004 ACC/AHA guidelines for the management of patients with ST-segment elevation MI are similar but recommend 75$162 \mathrm{mg}$ daily as maintenance therapy after ST-segment elevation MI. Aspirin therapy is considered a class I recommendation (evidence supports that treatment is useful and effective) for all acute coronary syndromes [16]. The initial dose of aspirin should be chewed and then swallowed during acute coronary syndromes to attain a rapid onset of action.

4.2. Secondary Prevention for Chronic Stable Angina. A subgroup analysis of the US Physicians' Health Study (PHS) of 333 men with chronic stable angina indicated that aspirin reduced the relative risk of acute MI by $87 \%(P<0.001)$ [26]. The Swedish Angina Pectoris Aspirin Trial involved 2035 patients and found a $34 \%$ relative risk reduction in the occurrence of a first MI over a four-year follow-up period in patients receiving $75 \mathrm{mg}$ of aspirin daily, compared with patients receiving placebo [27].

The 2002 ACC/AHA guidelines for chronic stable angina include a class IIa recommendation (the weight of evidence where opinion is in favor of usefulness and efficacy) for prophylactic aspirin therapy to prevent MI and death [28].

4.3. Secondary Prevention for Revascularization. Aspirin has been widely accepted as a cornerstone therapy in reducing ischemic complications of coronary revascularization with either coronary artery bypass graft surgery, balloon angioplasty, or stent implantation [29-31]. A number of studies have demonstrated the efficacy of aspirin in preventing thrombosis, a common event following revascularization [32-35].
Aspirin administered in the immediate postoperative period following bypass surgery decreases the rate of graft occlusion by approximately 50\%, and continued therapy leads to further decreases [29, 34]. Use of aspirin before and after coronary intervention is essential in the prevention of thrombosis. Early trials indicated that, in patients undergoing PCI, aspirin reduced mortality, MI, urgent revascularization, or stent thrombosis both with and without thienopyridines [18, 36-38].

The 2004 ACC/AHA guidelines for coronary artery bypass graft surgery suggest daily aspirin therapy with 100$325 \mathrm{mg}$ started within 24 hours after surgery [39]. The 2005 ACC/AHA guidelines for percutaneous coronary intervention recommend 75-325 mg of aspirin before the PCI procedure is performed in patients already taking daily chronic aspirin therapy, and 300-325 mg of aspirin at least 2 hours and preferably 24 hours before the PCI procedure is performed in patients not already taking daily chronic aspirin therapy [40]. After the PCI procedure, in patients with neither aspirin resistance, allergy, nor increased risk of bleeding, aspirin $162-325 \mathrm{mg}$ daily should be given for at least 1 month after BMS implantation, 3 months after sirolimuseluting stent implantation, and 6 months after paclitaxeleluting stent implantation, after which daily chronic aspirin use should be continued indefinitely at a dose of 75 to $162 \mathrm{mg}$ [40]. All of these recommendations belong to class I (evidence supports that treatment is useful and effective).

4.4. Secondary Prevention for Stroke and Transient Ischemic Attack. The previous meta-analysis by the ATT Collaboration involved 18270 patients with a history of stroke or transient ischaemic attack in 21 trials [17]. The result showed that antiplatelet therapy (mainly aspirin alone) for a mean duration of 29 months can significantly reduce the rate of major vascular events by $22 \%$. Treating 1000 patients with a history of cerebrovascular disease for this duration will prevent about 36 vascular events, mostly nonfatal stroke recurrence (25 fewer per 1000 treated), and some nonfatal myocardial infarction (5 fewer per 1000).

4.5. Secondary Prevention for Atrial Fibrillation. The presence of atrial fibrillation (AF) gives rise to the development of atrial thrombus and consequently increases the risk of stroke among elderly people. Vitamin K antagonists, most notable among which is warfarin, significantly reduce the risk of stroke by almost two-thirds compared to placebo. Owing to the difficulties with using warfarin of its requirement for frequent monitoring of the INR and increased hemorrhagic risk with increased duration of therapy, aspirin has been considered a potential alternate $[41-43]$.

Most of the evidence about the effects of aspirin therapy among patients with atrial fibrillation was provided by the European atrial fibrillation trial [44]. High-risk patients with a previous stroke or transient ischemic attack were randomized to aspirin or placebo (or oral anticoagulant, if eligible) in this trial. It was indicated that aspirin is a safe, though less effective, alternative when anticoagulation is contraindicated. Aspirin prevents 40 vascular events for 
every 1,000 treated patients. The previous meta-analysis by the ATT Collaboration [17] included 2770 patients with atrial fibrillation in four trials and found that there was a proportional reduction of $24 \%$ (9\%) in serious vascular events.

In patients with "lone AF", who are under 65 years of age, not hypertensive, without evidence of cardiovascular disease and who have normal echocardiograms, the baseline stroke risk of this cohort is relatively low (approximately $0.5 \%$ /year). In this situation, aspirin alone is considered by most experts to be adequate [41-43].

\section{Primary Prevention}

For primary prevention, the balance between benefits and risks of aspirin use is less clear because the absolute benefits of aspirin are generally lower than those in secondary prevention. Current guidelines largely ignore any differences in bleeding risk and recommend that aspirin be used widely for primary prevention in those at moderately raised risk of coronary heart disease. It has also been suggested that, since age is a major determinant of the risk of coronary heart disease, daily aspirin should be started in all people above a specific age, either alone or in combination with other drugs.

To date, six completed randomized trials have evaluated the benefits and risks of low-dose aspirin for the primary prevention of cardiovascular disease. The British Male Doctors' Trial (BDT) [45] of 5139 male physicians and the US Physicians' Health Study (PHS) [26] of 22071 healthy male were completed during the late 1980s. The Thrombosis Prevention Trial (TPT) [46] of 5085 men and the Hypertension Optimal Treatment (HOT) [47] trial of 18790 (47\% women) patients were completed in 1998. The Primary Prevention Project (PPP) [48] study of 4495 (58\% women) patients and the Women's Health Study (WHS) [49] of 39876 healthy females were completed in the 2000s. In all these trials patients were randomized to aspirin and had follow-up durations ranging from 3.6 to 10.1 years. The PHS and BDT used aspirin regimens of $325 \mathrm{mg}$ every other day and $500 \mathrm{mg} /$ day, respectively, whereas the TPT and HOT used $75 \mathrm{mg} /$ day of aspirin and the PPP and WHS used $100 \mathrm{mg} /$ day of enteric-coated aspirin.

The Antithrombotic Trialists' (ATT) Collaboration undertook a meta-analysis in the 6 previous trials and found that, in the primary prevention trials, aspirin use yielded a $12 \%$ proportional reduction in serious vascular events ( $0.51 \%$ aspirin versus $0.57 \%$ control per year, $P=0.0001$ ), due mainly to about $20 \%$ reduction in nonfatal myocardial infarction $(0.18 \%$ versus $0.23 \%$ per year, $P<0.0001)$ [23]. The net effect on stroke was not significant $(0.20 \%$ versus $0.21 \%$ per year, $P=0.4$ : haemorrhagic stroke $0.04 \%$ versus $0.03 \%, P=0.05$; other strokes $0.16 \%$ versus $0.18 \%$ per year, $P=0.08)$. Vascular mortality did not differ significantly $(0.19 \%$ versus $0.19 \%$ per year, $P=0.7)$. Aspirin use increased major gastrointestinal and extracranial bleeds $(0.10 \%$ versus $0.07 \%$ per year, $P<0.0001$ ), and the main risk factors for coronary disease were also risk factors for bleeding.

To better understand the impact of sex on the response to aspirin, Berger and colleagues conducted a meta-analysis on the sex-specific benefits of aspirin in 51342 women and
44114 men enrolled in the 6 previous prevention trials [50]. The results demonstrate that aspirin therapy is associated with a significant reduction in the risk of cardiovascular events in both sexes. However, the specific types of benefit differ in important ways between women and men. Aspirin use in women was associated with statistically significant reductions in cardiovascular events (odds ratio [OR], 0.88 [CI, 0.79 to 0.99$]$ ) and ischemic strokes (OR, 0.76 [CI, 0.63 to 0.93$]$ ); no statistically significant benefit was found in the reduction of myocardial infarctions or cardiovascular mortality. In men, aspirin use was associated with a statistically significant reduction in cardiovascular events $(\mathrm{OR}, 0.86$ [CI, 0.78 to 0.94$]$ ) and myocardial infarctions (OR, 0.68 [CI, 0.54 to 0.86$]$ ); no statistically significant benefit was found in the reduction of ischemic strokes or cardiovascular mortality. Total mortality was not significantly reduced by aspirin use in men or women.

In summary, consistent evidence from randomized clinical trials indicates that aspirin use reduces the risk for CVD events in adults without a history of CVD. For primary prevention of cardiovascular disease, aspirin therapy significantly reduced the risk of the composite of cardiovascular events primarily by reducing the risk of ischemic stroke with no significant effect on the risk of MI in women and predominantly by reducing the risk of MI with no significant effect on the risk of stroke in men.

\section{Adverse Effects}

Aspirin prevents thrombotic events by inhibiting prostaglandin synthesis, which also leads to adverse side effects, mainly including upper-gastrointestinal (GI) toxicity, extracranial and intracranial haemorrhage [51-53].

Aspirin-induced GI toxicity detected in randomized clinical trials, including nausea, heartburn, and epigastric pain, appears to be dose related in the range of 30 to $1,300 \mathrm{mg} / \mathrm{d}$. The principle mechanism is due to the inhibition of COX-1-dependent prostaglandin E2 (PGE2) synthesis by aspirin, while PGE2 inhibits acid secretion in gastric mucosa and increases mucous formation. Buffered and entericcoated aspirin preparations developed to attenuate local gastric erosion and minimize this side effect [51].

The overall risk of major extracranial and intracranial hemorrhage associated with antiplatelet drugs is difficult to assess in individual trials because their incidence is low $[52,53]$. In the overview of the ATT Collaboration [17], the overall proportional increase in risk of a major extracranial hemorrhage with aspirin therapy was about one-half (odds ratio $[\mathrm{OR}], 1.6 ; 95 \% \mathrm{CI}, 1.4$ to 1.8 ). After allowing for noncompliance in the trials, they are compatible with the 2 - to 2.5 -fold excess observed in case-control studies. The overall absolute excess of intracranial hemorrhage due to aspirin therapy was $<1$ per 1000 patients per year in highrisk trials, with somewhat higher risks in patients with cerebrovascular disease.

Moreover, chronic large dose of aspirin use may reduce renal blood flow and glomerular filtration and impair renal function due to the inhibition of COX-2-dependent PGI2, which support renal perfusion, diminish vascular resistance, 
and facilitate natriuresis [54]. This side effect often occurs at high aspirin doses and most frequently in elderly patients and those with established renal disease.

Furthermore, high-dose aspirin may also attenuate the benefit of angiotensin-converting enzyme (ACE) inhibitors in hypertensive and congestive heart failure patients because aspirin may attenuate the synthesis of PGE3 and PGI2, which is promoted by ACE inhibitors [55-57].

In summary, aspirin is effective for the prevention of thrombosis because of the inhibition of TXA2-dependent platelet function, which is also associated with excess bleeding. Assessing the net effect requires an estimation of the absolute thrombotic versus hemorrhagic risk of the individual patient.

\section{Aspirin Resistance}

Aspirin resistance has been used to describe the inability of aspirin to protect individuals from thrombotic complications, cause a prolongation of the bleeding time, reduce TXA2 production, or produce typical effect in vitro tests of platelet function $[58,59]$. However, a standard, clear, and distinct definition of aspirin resistance has not been established yet.

The rate of aspirin resistance is widely variable, ranging from 5 to $60 \%$ of the population affected by cardiovascular and cerebrovascular diseases in different studies [58-60]. It is difficult to know the exact prevalence of aspirin resistance from these studies because of variabilities in definitions for aspirin resistance, variabilities in testing and measurement between studies, small sample size of the studies, and different populations used in the studies. Many laboratory tests are currently used to investigate platelet activity and platelet response to aspirin, such as measurements of thromboxane biosynthesis, platelet aggregation, and platelet activation, bleeding time.

The potential mechanisms of aspirin resistance include enhanced platelet turnover, genetic polymorphisms of COX1 and other genes involved in thromboxane biosynthesis, upregulation of nonplatelet sources of thromboxane biosynthesis, and the interactions of other drugs $[58,61,62]$.

Because of a series of adverse cardiovascular events associated with aspirin resistance, once aspirin resistance is confirmed by laboratory measures, recommendations for alteration of therapy (dose change or additional antiplatelet agent) and followup are needed for meaningful clinical outcomes.

\section{Conclusions}

Aspirin remains the cornerstone of antiplatelet therapy in patients with cardiovascular disease. It decreases mortality and recurrence of cardiovascular events when used as acute therapy following acute coronary syndrome, thrombotic stroke, and Kawasaki's disease. It is also of proven benefit in secondary prevention among a wide range of patients, including those with acute coronary syndrome, stable angina, revascularization, stroke, TIA, and atrial fibrillation. In primary prevention, aspirin therapy appears to reduce the risk for CVD events in adults without a history of CVD with sex specific benefits. Aspirin may be considered for patients with a high risk of future CVD, but the benefits must be weighed against the possibility of side effects. The concept of resistance to aspirin is still an emerging and important clinical question requiring further study.

\section{References}

[1] A. Chockalingam, I. Balaguer-Vintro, A. Achutti et al., "The world heart federation's white book: impending global pandemic of cardiovascular diseases: challenges and opportunities for the prevention and control of cardiovascular diseases in developing countries and economies in transition," Canadian Journal of Cardiology, vol. 16, no. 2, pp. 227-229, 2000.

[2] C. H. Hennekens, M. L. Dyken, and V. Fuster, "Aspirin as a therapeutic agent in cardiovascular disease: a statement for healthcare professionals from the American heart association," Circulation, vol. 96, no. 8, pp. 2751-2753, 1997.

[3] J. R. Vane, "Inhibition of prostaglandin synthesis as a mechanism of action for aspirin-like drugs," Nature, vol. 231, no. 25, pp. 232-235, 1971.

[4] J. L. Masferrer, B. S. Zweifel, K. Seibert, and P. Needleman, "Selective regulation of cellular cyclooxygenase by dexamethasone and endotoxin in mice," Journal of Clinical Investigation, vol. 86, no. 4, pp. 1375-1379, 1990.

[5] D. A. Kujubu, B. S. Fletcher, B. C. Varnum, R. W. Lim, and H. R. Herschman, "TIS10, a phorbol ester tumor promoterinducible mRNA from Swiss 3T3 cells, encodes a novel prostaglandin synthase/cyclooxygenase homologue," Journal of Biological Chemistry, vol. 266, no. 20, pp. 12866-12872, 1991.

[6] J. R. Vane, R. J. Flower, and R. M. Botting, "History of aspirin and its mechanism of action," Stroke, vol. 21, supplement 12, pp. IV12-IV23, 1990.

[7] V. Fuster, L. Badimon, J. J. Badimon, and J. H. Chesebro, "The pathogenesis of coronary artery disease and the acute coronary syndromes," New England Journal of Medicine, vol. 326, no. 4, pp. 242-250, 1992.

[8] J. R. Vane and R. M. Botting, "The mechanism of action of aspirin," Thrombosis Research, vol. 110, no. 5-6, pp. 255-258, 2003.

[9] S. Husain, N. P. Andrews, D. Mulcahy, J. A. Panza, and A. A. Quyyumi, "Aspirin improves endothelial dysfunction in atherosclerosis," Circulation, vol. 97, no. 8, pp. 716-720, 1998.

[10] P. M. Ridker, M. Cushman, M. J. Stampfer, R. P. Tracy, and C. H. Hennekens, "Inflammation, aspirin, and the risk of cardiovascular disease in apparently healthy men," New England Journal of Medicine, vol. 336, no. 14, pp. 973-979, 1997.

[11] K. A. Steer, T. M. Wallace, C. H. Bolton, and M. Hartog, "Aspirin protects low density lipoprotein from oxidative modification," Heart, vol. 77, no. 4, pp. 333-337, 1997.

[12] "Randomised trial of intravenous streptokinase, oral aspirin, both, or neither among 17,187 cases of suspected acute myocardial infarction: ISIS-2. ISIS-2 (Second International Study of Infarct Survival) Collaborative Group," The Lancet, vol. 2, no. 8607, pp. 349-360, 1988.

[13] H. D. Lewis Jr., J. W. Davis, D. G. Archibald Jr. et al., "Protective effects of aspirin against acute myocardial infarction and death in mean with unstable angina. Results of a Veterans Administration Cooperative Study," New England Journal of Medicine, vol. 309, no. 7, pp. 396-403, 1983. 
[14] J. A. Cairns, M. Gent, J. Singer et al., "Aspirin, sulfinpyrazone, or both in unstable angina. Results of a Canadian Multicenter Trial," New England Journal of Medicine, vol. 313, no. 22, pp. 1369-1375, 1985.

[15] J. L. Anderson, C. D. Adams, E. M. Antman et al., "ACC/AHA 2007 Guidelines for the Management of Patients With Unstable Angina/Non-ST-Elevation Myocardial Infarction. A Report of the American College of Cardiology/American Heart Association Task Force on Practice Guidelines (Writing Committee to Revise the 2002 Guidelines for the Management of Patients With Unstable Angina/Non-ST-Elevation Myocardial Infarction) developed in collaboration with the American College of Emergency Physicians, the Society for Cardiovascular Angiography and Interventions, and the Society of Thoracic Surgeons endorsed by the American Association of Cardiovascular and Pulmonary Rehabilitation and the Society for Academic Emergency Medicine," Journal of the American College of Cardiology, vol. 50, no. 7, pp. e1-e157, 2007.

[16] E. M. Antman, D. T. Anbe, P. W. Armstrong et al., "ACC/AHA guidelines for the management of patients with ST-elevation myocardial infarction-executive summary: a report of the American College of Cardiology/American Heart Association Task Force on Practice Guidelines (writing committee to revise the 1999 guidelines for the management of patients with acute myocardial infarction)," Circulation, vol. 110, no. 5, pp. 588636, 2004.

[17] C. Baigent, C. Sudlow, R. Collins, and R. Peto, "Collaborative meta-analysis of randomised trials of antiplatelet therapy for prevention of death, myocardial infarction, and stroke in high risk patients," British Medical Journal, vol. 324, no. 7329, pp. 71-86, 2002.

[18] L. Schwartz, M. G. Bourassa, J. Lesperance et al., "Aspirin and dipyridamole in the prevention of restenosis after percutaneous transluminal coronary angioplasty," New England Journal of Medicine, vol. 318, no. 26, pp. 1714-1719, 1988.

[19] J. W. Newburger, M. Takahashi, M. A. Gerber et al., "Diagnosis, treatment, and long-term management of Kawasaki disease: a statement for health professionals from the Committee on Rheumatic Fever, Endocarditis and Kawasaki Disease, Council on Cardiovascular Disease in the Young, American Heart Association," Circulation, vol. 110, no. 17, pp. 27472771, 2004.

[20] P. Monagle, A. Chan, P. Massicotte, E. Chalmers, and A. D. Michelson, "Antithrombotic therapy in children: the seventh ACCP Conference on Antithrombotic and Thrombolytic Therapy," Chest, vol. 126, supplement 3, pp. 6455-6875, 2004.

[21] P. A.G. Sandercock, "The International Stroke Trial (IST): a randomised trial of aspirin, subcutaneous heparin, both, or neither among 19435 patients with acute ischaemic stroke," The Lancet, vol. 349, no. 9065, pp. 1569-1581, 1997.

[22] Z. -M. Chen, "CAST: randomised placebo-controlled trial of early aspirin use in 20,000 patients with acute ischaemic stroke," The Lancet, vol. 349, no. 9066, pp. 1641-1649, 1997.

[23] Antithrombotic Trialists' (ATT) Collaboration, "Aspirin in the primary and secondary prevention of vascular disease: collaborative meta-analysis of individual participant data from randomised trials," The Lancet, vol. 373, no. 9678, pp. 1849-1860, 2009.

[24] P. Théroux, H. Ouimet, J. McCans et al., "Aspirin, heparin, or both to treat acute unstable angina," New England Journal of Medicine, vol. 319, no. 17, pp. 1105-1111, 1988.

[25] L. C. Wallentin, U. Berglund, I. Nyman et al., "Aspirin $(75 \mathrm{mg} /$ day $)$ after an episode of unstable coronary artery disease: long-term effects on the risk for myocardial infarction, occurrence of severe angina and the need for revascularization," Journal of the American College of Cardiology, vol. 18, no. 7, pp. 1587-1593, 1991.

[26] T. J. Hartney, S. Shapiro, K. M. Jain et al., “The physicians' health study: aspirin for the primary prevention of myocardial infarction," New England Journal of Medicine, vol. 318, no. 14, pp. 924-926, 1988.

[27] S. Jull-Möller, N. Edvardsson, B. Jahnmatz, A. Rosen, S. Sorensen, and R. Omblus, "Double-blind trial of aspirin in primary prevention of myocardial infarction in patients with stable chronic angina pectoris," The Lancet, vol. 340, no. 8833, pp. 1421-1425, 1992.

[28] R. J. Gibbons, J. Abrams, K. Chatterjee et al., "ACC/AHA 2002 guideline update for the management of patients with chronic stable angina-summary article: a report of the American College of Cardiology/American Heart Association Task Force on Practice Guidelines (Committee on the Management of Patients With Chronic Stable Angina)," Circulation, vol. 107, no. 1, pp. 149-158, 2003.

[29] S. Goldman, J. Copeland, T. Mortiz et al., "Improvement in early saphenous vein graft patency after coronary artery bypass surgery with antiplatelet therapy: results of a Veterans Administration Cooperative Study," Circulation, vol. 77, no. 6, pp. 1324-1332, 1988.

[30] T. J. Gluckman, R. C. McLean, S. P. Schulman et al., "Effects of aspirin responsiveness and platelet reactivity on early vein graft thrombosis after coronary artery bypass graft surgery," Journal of the American College of Cardiology, vol. 57, no. 9, pp. 1069-1077, 2011.

[31] N. Kubota, T. Kasai, K. Miyauchi, W. Njaman, K. Kajimoto, and Y. Akimoto, "Therapy with statins and aspirin enhances long-term outcome of percutaneous coronary intervention," Heart and Vessels, vol. 23, no. 1, pp. 35-39, 2008.

[32] W. Njaman, K. Miyauchi, T. Kasai et al., "Impact of aspirin treatment on long-term outcome (over 10 years) after percutaneous coronary intervention," International Heart Journal, vol. 47, no. 1, pp. 37-45, 2006.

[33] K. A. A. Fox, S. R. Mehta, R. Peters et al., "Benefits and risks of the combination of clopidogrel and aspirin in patients undergoing surgical revascularization for non- ST-elevation acute coronary syndrome: the clopidogrel in unstable angina to prevent recurrent ischemic events (CURE) trial," Circulation, vol. 110, no. 10, pp. 1202-1208, 2004.

[34] T. P. Gavaghan, V. Gebski, and D. W. Baron, "Immediate postoperative aspirin improves vein graft patency early and late after coronary artery bypass graft surgery: a placebocontrolled, randomized study," Circulation, vol. 83, no. 5, pp. 1526-1533, 1991.

[35] V. A. Ferraris, S. P. Ferraris, D. J. Moliterno et al., "The society of thoracic surgeons practice guideline series: aspirin and other antiplatelet agents during operative coronary revascularization (executive summary)," Annals of Thoracic Surgery, vol. 79, no. 4, pp. 1454-1461, 2005.

[36] R. R. Taylor, F. A. Gibbons, G. D. Cope, G. N. Cumpston, G. C. Mews, and P. Luke, "Effects of low-dose aspirin on restenosis after coronary angioplasty," American Journal of Cardiology, vol. 68, no. 9, pp. 874-878, 1991.

[37] N. J. Lembo, A. J. R. Black, G. S. Roubin et al., "Effect of pretreatment with aspirin versus aspirin plus dipyridamole on frequency and type of acute complications of percutaneous transluminal coronary angioplasty," American Journal of Cardiology, vol. 65, no. 7, pp. 422-426, 1990.

[38] E. S. Barnathan, J. S. Schwartz, and L. Taylor, "Aspirin and dipyridamole in the prevention of acute coronary thrombosis 
complicating coronary angioplasty," Circulation, vol. 76, no. 1, pp. 125-134, 1987.

[39] K. A. Eagle, R. A. Guyton, R. Davidoff et al., "ACC/AHA 2004 guideline update for coronary artery bypass graft surgery: a report of the American College of Cardiology/American Heart Association Task Force on Practice Guidelines (Committee to Update the 1999 Guidelines for Coronary Artery Bypass Graft Surgery)," Circulation, vol. 110, no. 14, pp. e340-e437, 2004.

[40] S. C. Smith Jr., T. E. Feldman, J. W. Hirshfeld Jr. et al., "ACC/AHA/SCAI 2005 guideline update for percutaneous coronary intervention: a report of the American College of Cardiology/American Heart Association Task Force on Practice Guidelines (ACC/AHA/SCAI Writing Committee to Update the 2001 Guidelines for Percutaneous Coronary Intervention)," Circulation, vol. 113, no. 7, pp. e166-e286, 2006.

[41] C. Medi, G. J. Hankey, and S. B. Freedman, "Stroke risk and antithrombotic strategies in atrial fibrillation,” Stroke, vol. 41, no. 11, pp. 2705-2713, 2010.

[42] L. Kalra and G. Y. H. Lip, "Antithrombotic treatment in atrial fibrillation," Heart, vol. 93, no. 1, pp. 39-44, 2007.

[43] D. E. Singer, G. W. Albers, J. E. Dalen, A. S. Go, J. L. Halperin, and W. J. Manning, "Antithrombotic therapy in atrial fibrillation: the Seventh ACCP Conference on Antithrombotic and Thrombolytic Therapy," Chest, vol. 126, no. 3, pp. 4295-4565, 2004.

[44] I. Dehaene, "European atrial fibrillation trial," Acta Neurologica Belgica, vol. 88, no. 3, pp. 172-173, 1988.

[45] R. Peto, R. Gray, R. Collins et al., "Randomised trial of prophylactic daily aspirin in British male doctors," British Medical Journal, vol. 296, no. 6618, pp. 313-316, 1988.

[46] T. W. Meade, H. C. Wilkes, C. C. Kelleher et al., "Thrombosis prevention trial: randomised trial of low-intensity oral anticoagulation with warfarin and low-dose aspirin in the primary prevention of ischaemic heart disease in men at increased risk," The Lancet, vol. 351, no. 9098, pp. 233-241, 1998.

[47] H. H. Parving, "Hypertension Optimal Treatment (HOT) trial," The Lancet, vol. 352, no. 9127, pp. 574-575, 1998.

[48] M. C. Roncaglioni, "Low-dose aspirin and vitamin E in people at cardiovascular risk: a randomised trial in general practice," Lancet, vol. 357, no. 9250, pp. 89-95, 2001.

[49] P. M. Ridker, N. R. Cook, I. M. Lee et al., "A randomized trial of low-dose aspirin in the primary prevention of cardiovascular disease in women," New England Journal of Medicine, vol. 352, no. 13, pp. 1293-1304, 2005.

[50] J. S. Berger, M. C. Roncaglioni, F. Avanzini, I. Pangrazzi, G. Tognoni, and D. L. Brown, "Aspirin for the primary prevention of cardiovascular events in women and men: a sex-specific meta-analysis of randomized controlled trials," Journal of the American Medical Association, vol. 295, no. 3, pp. 306-316, 2006.

[51] P. J. Roderick, H. C. Wilkes, and T. E. Meade, "The gastrointestinal toxicity of aspirin: an overview of randomised controlled trials," British Journal of Clinical Pharmacology, vol. 35, no. 3, pp. 219-226, 1993.

[52] S. Derry and Y. K. Loke, "Risk of gastrointestinal haemorrhage with long term use of aspirin: meta-analysis," British Medical Journal, vol. 321, no. 7270, pp. 1183-1187, 2000.

[53] J. He, P. K. Whelton, B. Vu, and M. J. Klag, "Aspirin and risk of hemorrhagic stroke. A meta-analysis of randomized controlled trials," Journal of the American Medical Association, vol. 280, no. 22, pp. 1930-1935, 1998.

[54] T. Juhlin, B. A. G. Jönsson, and P. Höglund, "Renal effects of aspirin are clearly dose-dependent and are of clinical importance from a dose of $160 \mathrm{mg}$," European Journal of Heart Failure, vol. 10, no. 9, pp. 892-898, 2008.

[55] K. J. Harjai, S. Solis, A. Prasad, and J. Loupe, "Use of aspirin in conjunction with angiotensin-converting enzyme inhibitors does not worsen long-term survival in heart failure," International Journal of Cardiology, vol. 88, no. 2-3, pp. 207214, 2003.

[56] M. H. Park, "Should aspirin be used with angiotensinconverting enzyme inhibitors in patients with chronic heart failure?" Congestive Heart Failure, vol. 9, no. 4, pp. 206-213, 2003.

[57] H. P. Brunner-La Rocca, "Interaction of angiotensin-converting enzyme inhibition and aspirin in congestive heart failure: long controversy finally resolved?" Chest, vol. 124, no. 4, pp. 1192-1194, 2003.

[58] A. Airee, H. M. Draper, and S. W. Finks, "Aspirin resistance: disparities and clinical implications," Pharmacotherapy, vol. 28, no. 8, pp. 999-1018, 2008.

[59] A. Papathanasiou, J. Guodevenos, and A. D. Tselepis, “Aspirin resistance in cardiovascular disease: pathogenesis, diagnosis and clinical impact," Current Pharmaceutical Design, vol. 15, no. 10, pp. 1085-1094, 2009.

[60] G. I. Lancaster, J. Srinivasan, and H. Jain, "Aspirin resistance: an update," Current Atherosclerosis Reports, vol. 11, no. 2, pp. 105-110, 2009.

[61] P. Blair and J. E. Freedman, "Aspirin resistance in atherosclerosis," Current Atherosclerosis Reports, vol. 10, no. 2, pp. 149-157, 2008.

[62] S. Tseeng and R. Arora, "Reviews: aspirin resistance: biological and clinical implications," Journal of Cardiovascular Pharmacology and Therapeutics, vol. 13, no. 1, pp. 5-12, 2008. 


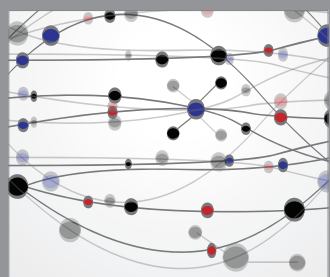

The Scientific World Journal
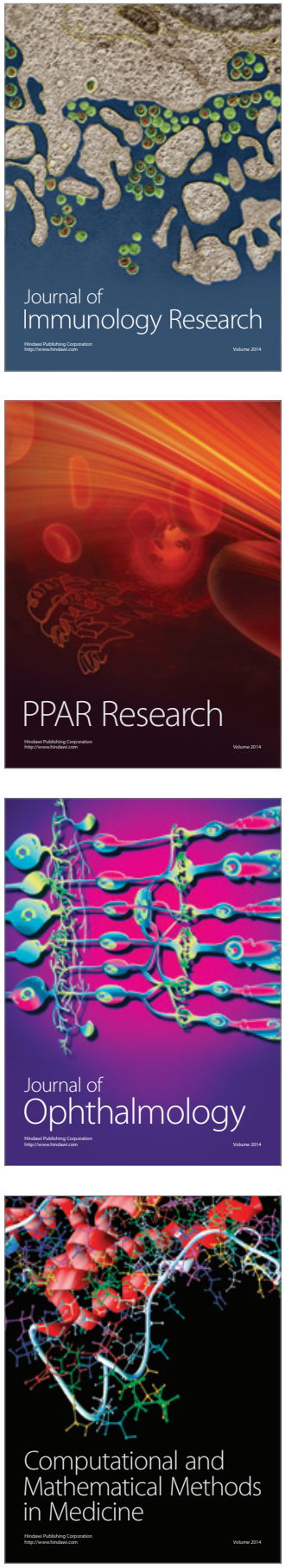

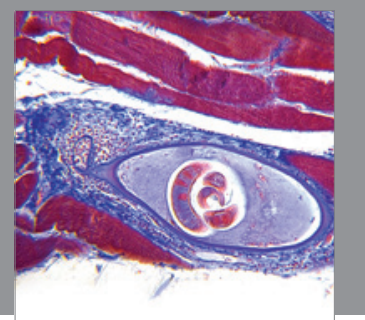

Gastroenterology

Research and Practice
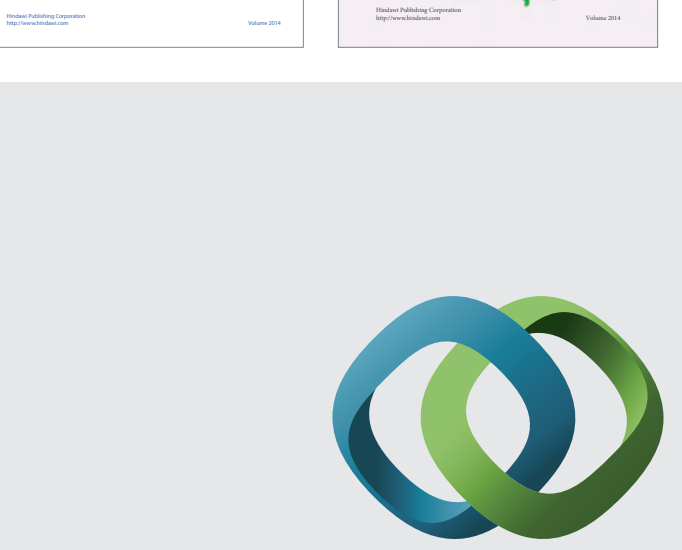

\section{Hindawi}

Submit your manuscripts at

http://www.hindawi.com
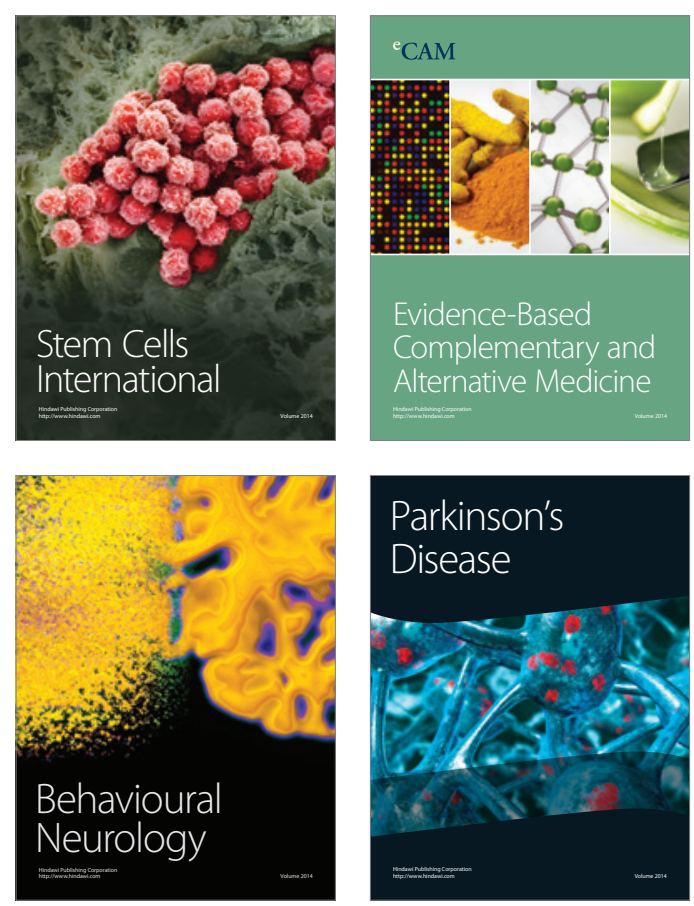

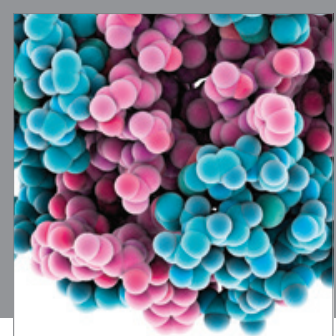

Journal of
Diabetes Research

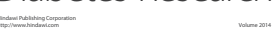

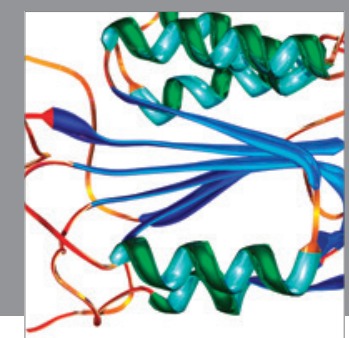

Disease Markers
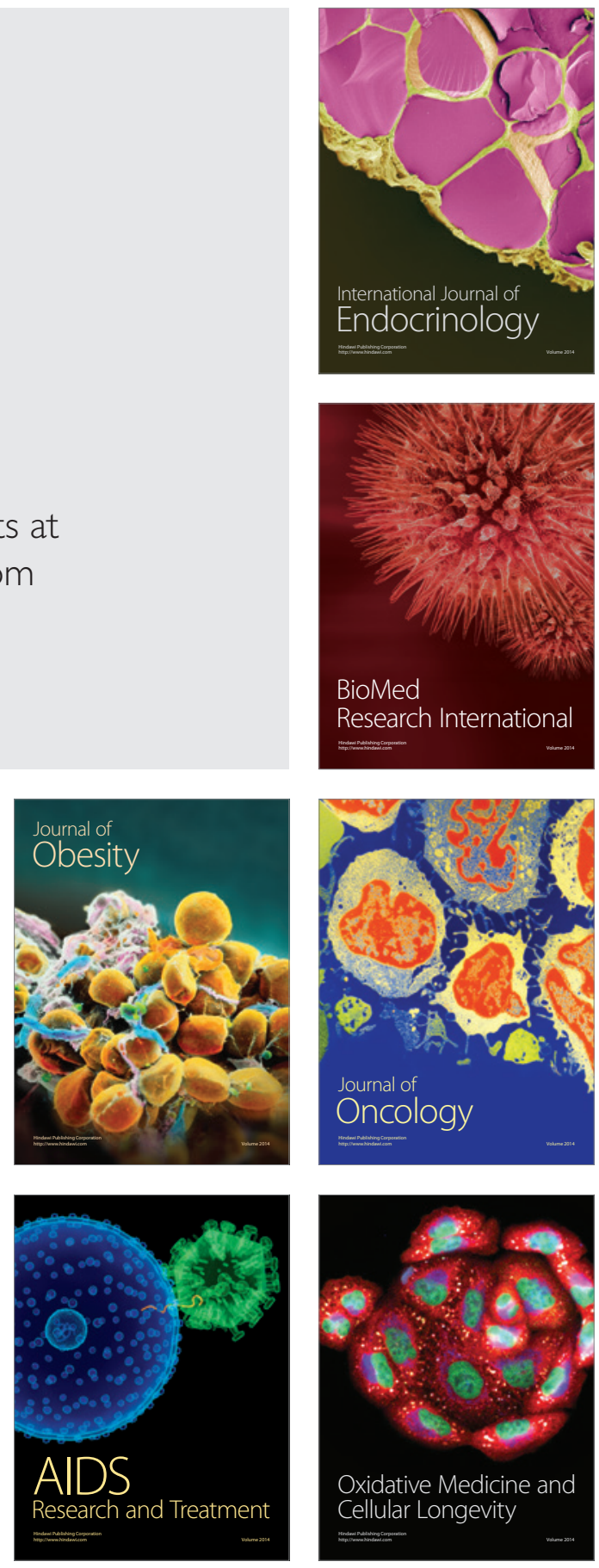